\title{
An Introduction to Instrumental Variables - Part 2: Mendelian Randomisation
}

\author{
Derrick A. Bennett \\ Clinical Trial Service Unit and Epidemiological Studies Unit, Nuffield Department of Clinical Medicine, \\ University of Oxford, Oxford, UK
}

\section{Key Words}

Mendelian randomisation - Instrumental variables analysis

\begin{abstract}
In the first part of this series, it was highlighted how even though randomised controlled trials can provide robust evidence for therapeutic interventions, for many types of exposure it may not be either practical or ethical to randomise patients to such studies (see part 1). Instrumental variables (IV) analyses have been increasingly employed in recent times in epidemiology to investigate the potential causal effects of an exposure. An IV is a variable that can realistically mimic the treatment allocation process in a randomised study and is assumed to be not directly related to outcome, except through the direct effect of treatment and not related to outcome through either measured or unmeasured confounders. As discussed in the first article, IV analyses can be useful in estimating direct treatment effects provided that the chosen instrument is strong. A particular type of IV analysis where a specific genetic variant has been used as the instrument known as 'Mendelian randomisation' has become increasingly common. The aim of the second part of this statistical primer is to outline the approach to Mendelian randomisation and some of the advantages and disadvantages of this approach.

Copyright $\odot 2010$ S. Karger AG, Basel
\end{abstract}

\section{What Is a Mendelian Randomisation Study?}

A Mendelian randomisation (MR) study has been described as essentially an instrumental variables (IV) analysis with a genetic instrument [1-3]. The fundamental principle of MR is that if there are genetic variants that can either alter the level of, or imitate the biological effects of, a potentially modifiable environmental exposure that itself has an effect on disease risk, then these genetic variants should be related to disease risk to the extent predicted by their influence on the exposure to the risk factor [4]. The name 'Mendelian randomisation' refers to the random assortment of genes (known as meiosis) transferred from parent to offspring at the time of gamete formation based on Mendel's second law [5]. Some of the advantages of an MR study are that genetically determined differences, if untreated, remain constant, are not influenced by selection bias and reflect prolonged differences and therefore are: (a) not susceptible to 'reverse causality'; (b) not influenced by confounding, and (c) independent of short-term fluctuations $[6,7]$. Genetic variants can influence circulating biochemical markers, such as cholesterol levels, and this fact means that it is possible to assess causality via associations observed between these biochemical markers or intermediate phenotypes (IP). If adequate control for population

\section{KARGER}

Fax +41613061234 E-Mail karger@karger.ch www.karger.com
Dr. Derrick A. Bennett

Clinical Trial Service Unit and Epidemiological Studies Unit

Nuffield Department of Clinical Medicine, University of Oxford

Richard Doll Building, Old Road Campus, Roosevelt Drive, Oxford OX3 7LF (UK)

Tel. +44 186574 3949, Fax +44 186574 3985, E-Mail derrick.bennett@ctsu.ox.ac.uk 
Fig. 1. MR as a form of IV analysis. Adapted from [4].

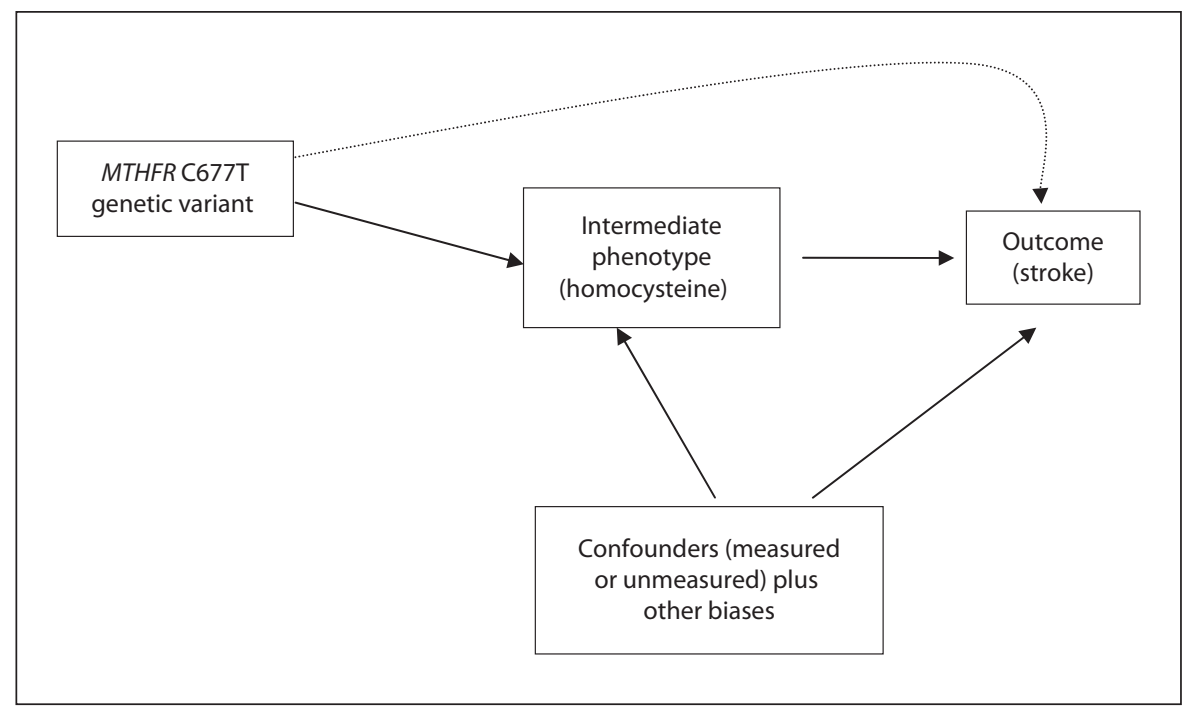

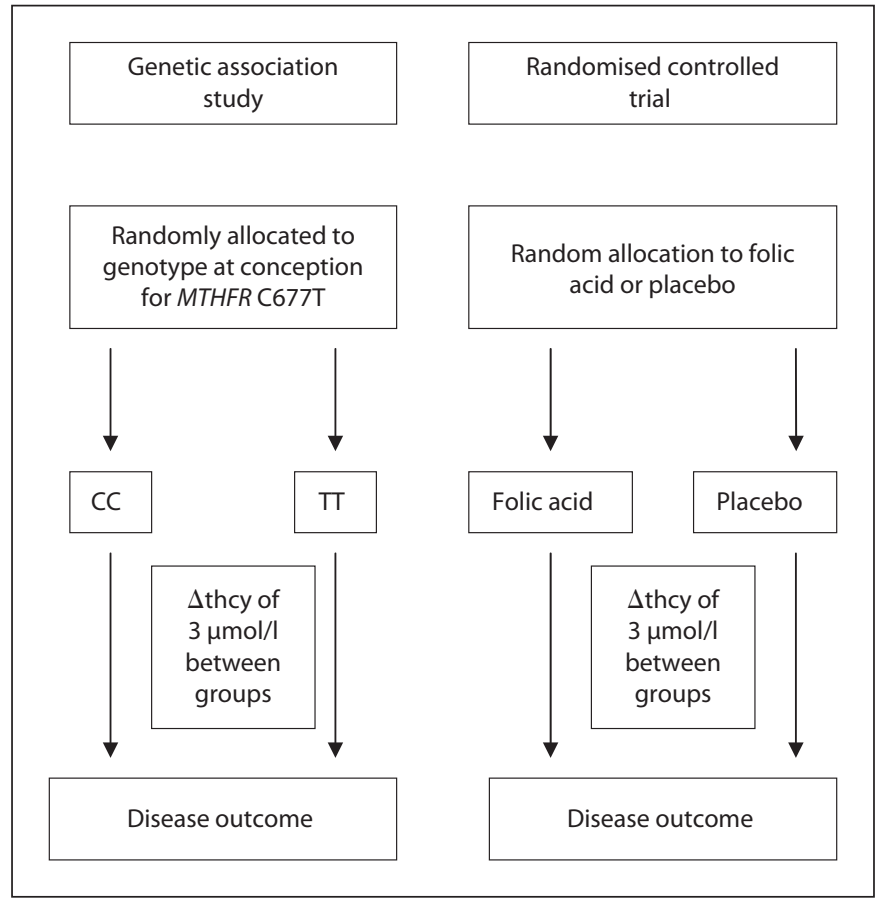

Fig. 2. Comparison of an MR study with a randomised controlled trial. thcy = Plasma total homocysteine. Adapted from [6].

stratification (see Panel for definition) has been performed then bias and confounding due to environmental factors is greatly reduced and MR studies could give an important indication on whether interventions to modify these IP could influence disease risk.

\section{Example of an MR Study}

Elevated plasma total homocysteine concentrations have been associated with risk of stroke in observational studies, but it is uncertain if these associations are causal $[8,9]$. Several studies have investigated the relationship between the $677 \mathrm{C} \rightarrow \mathrm{T}$ polymorphism for the methylenetetrahydrofolate reductase (MTHFR) gene (that is associated with elevated homocysteine concentrations) and stroke using an MR approach to the analysis.

Figure 1 presents the basic structure of an MR approach to analysis in using the MTHFR C677T polymorphism as an IV. The dotted line between the genetic variant and the outcome provides an estimate of the causal association between the IP that the genetic variant is a proxy for and the outcome. Individuals with the TT genotype have a higher level of plasma homocysteine than those with the CC genotype, a difference of approximately $3 \mu \mathrm{mol} / \mathrm{l}$. Homocysteine levels are known to be lowered by folic acid by about $25 \%$, which is equivalent to a $3 \mu \mathrm{mol} / \mathrm{l}$ difference. Randomised controlled trials that have aimed to assess whether lowering homocysteine by administering folic acid reduces the risk of stroke compared to placebo have been inconclusive $[10,11]$ possibly because of mandatory and voluntary fortification of folic acid in foods in some countries but not others. Figure 2 demonstrates how the MR approach can be seen as being analogous to a randomised controlled trial in this situation.

In an MR study by Alluri et al. [12], the authors found that the prevalences of CT and TT genotypes in patients 
with arterial stroke were 30 and $1.4 \%$ compared to 2.04 and $0 \%$ in controls. Patients with arterial stroke had homocysteine values almost twice as large as controls. The authors suggested that MTHFR evaluation may help in preventing or reducing the morbidity caused by stroke.

\section{Limitations of MR Studies}

There are several limitations of MR studies that are described in extensive detail elsewhere [4, 13-16]. Briefly, it is extremely important to ensure that there is a strong relationship between the genetic variant and the IP (e.g. a biochemical marker) to estimate the effects of the IP on the outcome. This requires that there is no biological adaptation or canalisation (see Panel for definition). When using a case-control design, which assesses the IP after determination of disease status classical IV estimates of the direct treatment effect would be invalid if the disease alters the IP, while the gene-disease association is unaffected by reverse-causality. Usual practice is to therefore examine the association between gene and biochemical marker in controls only. A gene may act via more than one pathway, a phenomenon known as pleiotropy (see Panel for definition). If pleioptropy occurs in IP then the use of IV may be confounded by other pathways leading from gene to outcome thus invalidating the assumption of the genetic variant being associated with outcome only via IP. If there is an inadequate understanding of the function of the genetic variants then pleiotropy could be a serious problem. Pleiotropy is unlikely to occur in an MR study if the genetic variant being used as the instrument and its effect on the IP has been extensively studied, as is the case

\section{Panel: Genetic-Specific Limitations of MR}

(1) Population stratification: confounding of the gene-disease association by ethnicity; allele frequencies, disease rates or exposure rates can have a large amount of variation between different subgroups of the population and can lead to spurious associations

(2) Canalisation: compensatory biological adaption to the effects on disease risk

(3) Pleiotropy: the effects of genetic polymorphisms on more than one biological system (i.e. a genetic variant may have effects on other intermediate phenotypes)

(4) Linkage disequilibrium: refers to a statistical association (or correlation) between genetic polymorphisms, usually because the polymorphisms are close together on the genome with the MTHFR C677T polymorphism. Pleiotropy is relatively common but will not violate the assumptions if the variant is associated with pleiotropic effects that do not influence the outcome. Genetic variants with pleiotropic effects that do influence the outcome of interest will invalidate the MR study [17]. Confounding of genotype by linkage disequilibrium (see Panel for definition) between the genetic variant of interest and another genetic variant that influences the outcome will invalidate the MR study. However, if the other genetic variant only affects the outcome via its effects on the same IP then the MR approach will still be valid.

It follows that in MR studies there will be some degree of bias for estimates of the direct treatment effect when the aforementioned assumptions are not met [18]. An individual association study that has been powered adequately to detect a genetic effect on the biochemical marker of interest may be underpowered to estimate genetic effects on disease. As a consequence, it is not possible to say with certainty whether the absence of an IP effect on outcome is due to the absence of a significant genetic effect on outcome. A common strategy to overcome this problem is to use meta-analyses of existing genetic association studies. Casas et al. [19] used meta-analysis to assess whether the association between homocysteine and stroke was potentially causal. They observed that there was an increase in the risk of stroke for individuals with the TT genotype of the MTHFR C677T variant that was compatible with the risk predicted from the association for homocysteine and stroke in conjunction with the differences in homocysteine seen between CC and TT genotypes. This provided evidence to support a causal link between homocysteine and stroke. However, genetic association meta-analyses can suffer from other biases such as publication bias, selection bias or environmental exposure measurement error. In addition, if estimates of gene-disease, gene-IP, and IP-disease associations come from different studies, it is possible that estimates could be prone to substantial bias, if different populations used different disease definitions.

Classical IV analyses assume linear relationships between the variables, but disease status is usually measured as a binary variable and analysed via logistic regression. When the assumption of linearity is violated, the IV estimates will be biased [20]. It is thus recommended that sensitivity analyses be performed to assess the robustness of the conclusions to different amounts of hypothesised confounding from unmeasured confounders and a range of likely values for assessment of IP-disease associations [21].

Neuroepidemiology 2010;35:307-310 


\section{Conclusion}

MR studies have become increasingly popular in assessing the potential effects of lowering the levels of a potentially causal biomarker. MR studies have been particularly successful in cardiovascular epidemiology with MR showing strong evidence of causality forlipoprotein(a) [22], demonstrating good evidence for a lack of causality of C-reactive protein [23] and fibrinogen [24]. MR can also be applied when the exposure is a modifiable behav- iour rather than an IP (e.g. alcohol intake) [25]. There is also an active area of methodological research that is investigating the use of multiple genetic variants as instruments in order to improve the estimates of direct treatment effects [26]. MR studies present an opportunity to neuroepidemiologists to identify potentially modifiable exposures which may be tested in future large-scale intervention studies that can assess reversibility and establish the precise magnitude of treatment of effects and any unexpected adverse effects of the intervention.

\section{References}

$\checkmark 1$ Bennett D: An introduction to instrumental variables analysis: Part 1. Neuroepidemiology 2010;35:237-240.

-2 Morshed S, Tornetta P 3rd, Bhandari M: Analysis of observational studies: a guide to understanding statistical methods. J Bone Joint Surg Am 2009;91(suppl 3):50-60.

$\checkmark 3$ Wehby GL, Ohsfeldt RL, Murray JC: 'Mendelian randomization' equals instrumental variable analysis with genetic instruments. Stat Med 2008;27:2745-2749.

4 Smith GD, Timpson N, Ebrahim S: Strengthening causal inference in cardiovascular epidemiology through Mendelian randomization. Ann Med 2008;40:524-541.

5 Morgan TH: Heredity and Sex. New York, Columbia University Press, 1913.

6 Davey Smith G, Ebrahim S: What can Mendelian randomisation tell us about modifiable behavioural and environmental exposures? BMJ 2005;330:1076-1079.

7 Davey Smith G, Ebrahim S, Lewis S, Hansell AL, Palmer LJ, Burton PR: Genetic epidemiology and public health: hope, hype, and future prospects. Lancet 2005;366:1484-1498.

$>8$ Verhoef P, Hennekens CH, Malinow MR, Kok FJ, Willett WC, Stampfer MJ: A prospective study of plasma homocyst(e)ine and risk of ischemic stroke. Stroke 1994;25:19241930.

$\checkmark 9$ Homocysteine Studies Collaboration: Homocysteine and risk of ischemic heart disease and stroke: a meta-analysis. JAMA 2002;288:2015-2022.

>10 Saposnik G, Ray JG, Sheridan P, McQueen M, Lonn E: Homocysteine-lowering therapy and stroke risk, severity, and disability: additional findings from the HOPE 2 trial. Stroke 2009;40:1365-1372.
11 Toole JF, Malinow MR, Chambless LE, et al: Lowering homocysteine in patients with ischemic stroke to prevent recurrent stroke, myocardial infarction, and death: the Vitamin Intervention for Stroke Prevention (VISP) randomized controlled trial. JAMA 2004;291:565-575.

12 Alluri RV, Mohan V, Komandur S, Chawda K, Chaudhuri JR, Hasan Q: MTHFR C677T gene mutation as a risk factor for arterial stroke: a hospital based study. Eur J Neurol 2005;12:40-44.

$>13$ Nitsch D, Molokhia M, Smeeth L, DeStavola BL, Whittaker JC, Leon DA: Limits to causal inference based on Mendelian randomization: a comparison with randomized controlled trials. Am J Epidemiol 2006;163:397403.

14 Sheehan NA, Didelez V, Burton PR, Tobin MD: Mendelian randomisation and causal inference in observational epidemiology. PLoS Med 2008;5:e177.

15 Didelez V, Sheehan N: Mendelian randomization as an instrumental variable approach to causal inference. Stat Methods Med Res 2007;16:309-330.

16 Thanassoulis G, O’Donnell CJ: Mendelian randomization: nature's randomized trial in the post-genome era. JAMA 2009;301:23862388.

17 Lawlor DA, Harbord RM, Sterne JA, Timpson N, Davey Smith G: Mendelian randomization: using genes as instruments for making causal inferences in epidemiology. Stat Med 2008;27:1133-1163.
18 Little J, Khoury MJ: Mendelian randomisation: a new spin or real progress? Lancet 2003;362:930-931.

19 Casas JP, Bautista LE, Smeeth L, Sharma P, Hingorani AD: Homocysteine and stroke: evidence on a causal link from Mendelian randomisation. Lancet 2005;365:224-232.

20 Palmer TM, Thompson JR, Tobin MD, Sheehan NA, Burton PR: Adjusting for bias and unmeasured confounding in Mendelian randomization studies with binary responses. Int J Epidemiol 2008;37:1161-1168.

21 Bautista LE, Smeeth L, Hingorani AD, Casas JP: Estimation of bias in nongenetic observational studies using 'Mendelian triangulation'. Ann Epidemiol 2006;16:675-680.

22 Kamstrup PR, Tybjaerg-Hansen A, Steffensen R, Nordestgaard BG: Genetically elevated lipoprotein(a) and increased risk of myocardial infarction. JAMA 2009;301: 2331-2339.

$>23$ Elliott P, Chambers JC, Zhang W, et al: Genetic loci associated with $\mathrm{C}$-reactive protein levels and risk of coronary heart disease. JAMA 2009;302:37-48.

-24 Keavney B, Danesh J, Parish S, et al: Fibrinogen and coronary heart disease: test of causality by 'Mendelian randomization'. Int J Epidemiol 2006;35:935-943.

$\checkmark 25$ Chen L, Davey Smith G, Harbord RM, Lewis SJ: Alcohol intake and blood pressure: a systematic review implementing a Mendelian randomization approach. PLoS Med 2008; 5:e52.

$>26$ Burgess S, Thompson SG, Burgess S, et al: Bayesian methods for meta-analysis of causal relationships estimated using genetic instrumental variables. Stat Med 2010;29: 1298-1311. 